\title{
CFD ANALYSIS OF LOW FREQUENCY OSCILLATIONS IN NEWTONIAN AND NON-NEWTONIAN FLUIDS IN A VERTICAL PIPE
}

\author{
MADURANGA AMARATUNGA, HERIMONJA A. RABENJAFIMANANTSOA \& RUNE W. TIME \\ Department of Energy and Petroleum Engineering, University of Stavanger, Norway
}

\begin{abstract}
The effect of vertically imposed, low frequency oscillations on the rheology of shear-thinning nonNewtonian fluids are studied numerically by computational fluid dynamics (CFD). Both Newtonian (water) and inelastic time-independent fluids of power-law (Poly-Anionic Cellulose (PAC)) are used as test fluids. Unsteady state simulations were performed using ANSYS Fluent version 18.0 for a vertical 2-D pipe geometry (ID $=50 \mathrm{~mm}$ and $\mathrm{H}=850 \mathrm{~mm}$ ) and sinusoidal, vertical oscillations to the liquid body itself were imposed with a user-defined function (UDF). The multiphase volume of fluid (VOF) method with realizable k- $\varepsilon$ method was used to impose the turbulence nature of the flow for the cases with water while the cases with non-Newtonian fluids were simulated under the laminar condition. Oscillations of different low frequency values $(0.25-5 \mathrm{~Hz})$ and different velocity amplitudes $(0.1-0.3 \mathrm{~m} / \mathrm{s})$ were tested numerically. The dynamic variation of velocity and shear rate within the oscillated, bulk liquid medium is demonstrated. The flow inside the vertical pipe acts plug like at higher frequencies for both Newtonian and non-Newtonian fluids. The air-liquid interface becomes unstable with small disruptive peaks for the cases with water at higher velocity amplitudes while that is very calm for the laminar cases with non-Newtonian fluids. The achieved velocity gradients and the resultant shear rate variation are low with the increased PAC concentration due to the viscous resistance. However, the instantaneous velocity profiles display a progressively more complex structure with increased frequency and velocity amplitude, revealing the presence of alternating upward/downward motion. These alternating velocity profiles confirm the varying shear field present within a drilling pipe at different frequencies and velocity amplitudes while the variation of the shear field is more dependent on the velocity amplitude.
\end{abstract}

Keywords: frequency, velocity amplitude, displacement amplitude, shear rate, dynamic velocity, Computational Fluid Dynamics.

\section{INTRODUCTION}

Drilling fluids may experience different frequencies and oscillations while they are circulating through the well. For an instance, in some cases, gas kicks may arise at underbalanced drilling conditions, where there is a possibility of severe oscillations and even extreme accelerations might take place until pressure equilibrium is reached by friction losses.

The shear-thinning behaviour of typical water-based drilling fluids contributes for a reduction in flow friction against the drill pipe walls. The apparent viscosity of such fluids decreases with the shear rate, which will eventually alter the dynamical behaviour of the drilling fluids. That is the main reason why the oscillatory motion should be considered so importantly when shear-thinning non-Newtonian drilling fluids are circulating through the well. Time and Rabenjafimanantsoa [1] describe this phenomenon further that, the oscillation frequency of such system is mainly related to mass inertia and to the gas compressibility, while the amplitude decay can be attributed to the liquid viscosity via friction. Since the shear rate and shear stress of drilling fluids may vary substantially over time under such scenarios, it is vital to design them accordingly to achieve desired solids carrying capacities and solids separation capacities. 
Understanding of dynamic velocity variation and thus the change of rate of shear within a vertically oscillating liquid body is hard due to complex rheology of the non-Newtonian fluid and its behaviour at different oscillating conditions. Computational Fluid Dynamics (CFD) modelling is an advanced tool to study the various aspects of dynamic velocity variations within such complex systems. In literature, studies can be found related to oscillatory flow in pipelines or simply in tubes [2]-[6]. However, almost all of them have investigated the flow enhancement or retardation when the liquid containing pipe is oscillated by different means and confirm that pulsatile flow of shear-thinning non-Newtonian fluids enhances the flow within pipelines [2]. Experimental and numerical study performed by Mena et al. [4] to examine the flow enhancement of viscoelastic fluids through pipes vibrated longitudinally reveals that the maximum enhancement achieved at maximum frequency and amplitude. But, the dominating factor in the oscillating flow of viscoelastic liquids in pipes is the shear dependent viscosity. According to the studies performed by Torralba et al. [7], it has been identified that, the remarkable enhancement in the dynamic response of the viscoelastic behaviour in the presence of a pulsating environment could be attributed to a resonant effect due to the elastic behaviour of the fluid and the geometry of the container as well.

However, the studies that address the effect of oscillatory motion on the rheology of nonNewtonian fluids are limited. Amaratunga et al. [8] have studied the impact of horizontal oscillation on non-Newtonian rheology in a square-cross sectional liquid column. Their efforts in quantification of the change of shear field close to the walls of the tank prove the fact that, the oscillatory motion which is experienced by shear-thinning non-Newtonian fluids will result in increased settling due to the reduction of flow resistance.

The objective of this study is to numerically investigate the effect of oscillation frequency and velocity amplitude on the dynamic velocity field within the liquid medium and to interpret its consequences on non-Newtonian rheology and settling of particles. The work is a part of the study, which aims to investigate the effect of vibration/oscillation on particle settling in shear-thinning non-Newtonian drilling fluids.

\section{COMPUTATIONAL MODEL AND METHODOLOGY}

Two-dimensional, two-phase CFD simulations were performed to investigate the effect of frequency and amplitude of oscillatory motion on the velocity field of a liquid column.

\subsection{The geometry, boundary conditions and material properties}

ANSYS DesignModeler 18.0 and ANSYS Meshing 18.0 were used for the geometry and mesh generation, respectively. A vertical pipe with an internal diameter of $50 \mathrm{~mm}$ and a length of $850 \mathrm{~mm}$ was used as shown in Fig. 1. A piston arrangement was made to provide the sinusoidal oscillatory motion to the liquid body itself and an air layer was considered on top of the liquid surface to study the interface behaviour during oscillations. Both Newtonian (water) and non-Newtonian fluids were simulated. For the non-Newtonian case $2 \mathrm{~g} / \mathrm{L}$ and $4 \mathrm{~g} / \mathrm{L}$ Poly-Anionic Cellulose (PAC) in water were used. The viscosity of the PAC solution was defined as a non-Newtonian power-law fluid in ANSYS Fluent.

A grid-independence study was carried out by performing number of simulations with different grid sizes to ensure satisfactory results without any influence on the grid size used. In this way, a grid size of $1 \mathrm{~mm}$ was selected as the maximum element size and the geometry was meshed with triangular cells. The outlet boundary was set as an "outflow" and the sidewalls were defined as deforming boundaries. The physical and rheological properties of the test fluids are listed in Table 1. The values for water and air are from Fluent data base and for PAC are from laboratory experiments. 


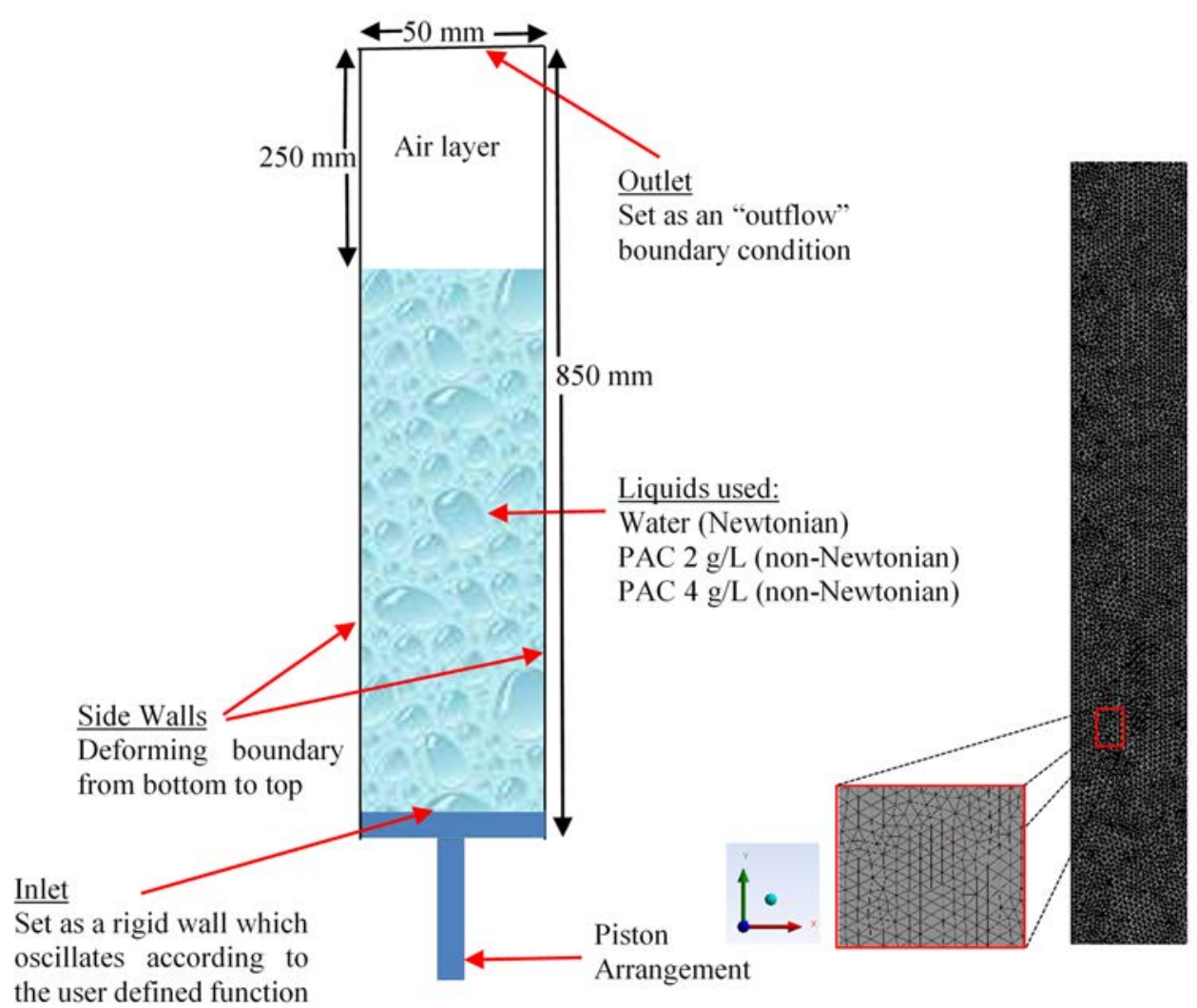

Figure 1: The geometry and the grid.

Table 1: Properties of the test materials.

\begin{tabular}{|l|c|c|c|c|c|c|}
\hline \multirow{2}{*}{ Material } & \multirow{2}{*}{$\begin{array}{c}\text { Density } \\
\left(\mathrm{kg} / \mathrm{m}^{3}\right)\end{array}$} & \multirow{2}{*}{\begin{tabular}{c} 
Newtonian \\
viscosity \\
\cline { 5 - 7 }
\end{tabular}} & & \multicolumn{4}{|c|}{$\begin{array}{c}\text { Nonsistency } \\
\text { index (K) }\end{array}$} & $\begin{array}{c}\text { Behaviour } \\
\text { index (n) } \\
\left(\mathrm{Pa} . \mathrm{s}^{\mathrm{n}}\right)\end{array}$ & $\begin{array}{c}\text { Minimum } \\
\text { viscosity } \\
\text { limit (Pa.s) }\end{array}$ & $\begin{array}{c}\text { Maximum } \\
\text { viscosity } \\
\text { limit (Pa.s) }\end{array}$ \\
\hline Water & 998.2 & 0.0010 & - & - & - & - \\
\hline Air & 1.225 & $1.79 \mathrm{e}-5$ & - & - & - & - \\
\hline PAC 2 g/L & 1000 & - & 0.057 & 0.78 & 0.013 & 0.036 \\
\hline PAC 4 g/L & 1000 & - & 0.194 & 0.71 & 0.024 & 0.124 \\
\hline
\end{tabular}

\subsection{CFD model}

The governing equations, which form the basis of the flow for a generalized incompressible Newtonian fluid in a pipe, can be written as [9]:

$$
\text { continuity } \quad \nabla . \boldsymbol{U}=0
$$




$$
\text { motion } \quad \rho \frac{D \boldsymbol{U}}{D t}=-\nabla \mathrm{p}+[\nabla \cdot \eta \dot{\gamma}]+\rho \boldsymbol{g},
$$

where, $\boldsymbol{U}$ is the velocity vector, $\mathrm{t}$ is the time, $\boldsymbol{g}$ is the gravitational acceleration. The term on the left-hand side of eqn (2) represents the mass per unit volume times acceleration, while the right-hand side represents the pressure force, viscous force and the gravity force per unit volume respectively. The non-Newtonian fluids considered in the simulations are of powerlaw type, i.e.:

$$
\eta=k \dot{\gamma}^{n-1},
$$

where, $\eta$ is the apparent viscosity, $\mathrm{k}$ is the consistency index, $\dot{\gamma}$ is the shear rate and $\mathrm{n}$ is the behaviour index of the non-Newtonian fluid. The transverse sinusoidal oscillatory motion was imposed on the liquid body itself mimicking a piston arrangement from the bottom in the y-direction normal to the pipe cross section as shown in Fig. 1. In this case of a simple harmonic motion, the displacement of the liquid body $y(t)$ from its middle position can be written by eqn (4):

$$
y(t)=a \sin (\omega t),
$$

where, $a$ is the piston displacement amplitude and $\omega=2 \pi f$. The time varying velocity $V(t)$ of the piston is simply the differentiation of eqn (4) and can be written as,

$$
V(t)=A \cos (\omega t)
$$

where $A=a \omega$. For an oscillatory flow, the oscillatory/vibrational Reynolds number $\left(\mathrm{Re}_{\mathrm{v}}\right)$ is defined as $\operatorname{Re}_{\mathrm{v}}=(\mathrm{a} \omega \mathrm{D} / \mathrm{v})$ [6], where $\mathrm{D}$ is the pipe diameter and $v$ is the kinematic viscosity of the fluid. It is the ratio between the forces of inertia and viscous forces in the boundary layer of the flow caused due to the oscillatory effect. For all the cases, the values of $\operatorname{Re}_{\mathrm{v}}$ were in the laminar regime $(<455)$ for both PAC fluids and in turbulent regime $(>9000)$ for water. The test conditions for the different simulation cases with different oscillation properties are given in Table 2.

Table 2: Conditions for the different simulation cases.

\begin{tabular}{|c|c|c|}
\hline Case \# & Frequency $(\mathrm{f})(\mathrm{Hz})$ & Velocity amplitude $(\mathrm{A})(\mathrm{m} / \mathrm{s})$ \\
\hline Case 1 & 0.25 & 0.1 \\
\hline Case 2 & 0.50 & 0.1 \\
\hline Case 3 & 0.75 & 0.1 \\
\hline Case 4 & 1.00 & 0.1 \\
\hline Case 5 & 5.00 & 0.1 \\
\hline Case 6 & 1.00 & 0.2 \\
\hline Case 7 & 1.00 & 0.3 \\
\hline
\end{tabular}

The inlet was defined as a "rigid body" in order to facilitate the provision of different properties (frequency and velocity amplitude) of the oscillatory motion using a user-defined function (UDF). The user-defined function was important to define the geometry and motion with time, since the zone adjacent to the inlet wall is deforming and moving at the same time.

The multiphase volume of fluid (VOF) method was employed to specify the air and fluid phases. The pressure based, realizable k- $\varepsilon$ turbulent model was used for all the cases with water and the test cases for non-Newtonian fluids were simulated under laminar conditions. 
Under the dynamic mesh settings, diffusion-based smoothing method with a diffusion parameter of 2 was utilized to update the volume mesh in the deforming regions.

All the simulation cases were solved over five oscillation periods in order to acquire more generalized result. It did not affect the accuracy of the result, but increased the simulation time in proportion. The time step size and the number of time steps were determined after a considerable amount of computational experimentation based on the frequency of the particular simulation case and solution convergence. The simulated liquid velocities and shear rates were extracted along a radial line located $200 \mathrm{~mm}$ below the initial liquid surface and displayed in Figs 3-7.

\section{RESULTS AND DISCUSSION}

The results of the 2-D transient simulations performed using ANSYS Fluent to investigate the effect of oscillatory motion on dynamic velocity and shear field are reported in this section. The displacement and the corresponding velocity of the piston motion at different positions within a complete phase cycle are illustrated in Fig. 2. In the following, the positions $\mathrm{A}, \mathrm{B}, \mathrm{C}$ and $\mathrm{D}$ refer to the different positions within a complete phase cycle as shown in Fig. 2.

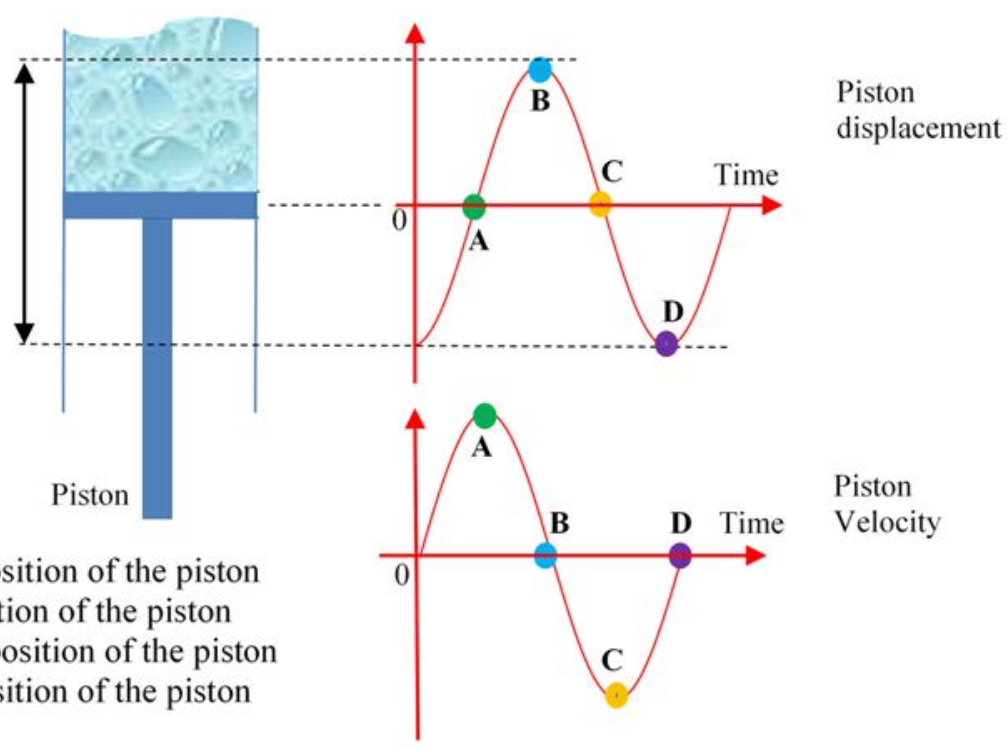

Figure 2: Displacement and velocity profiles of piston arrangement at different phase positions.

\subsection{Effect of oscillatory motion on air-liquid interface behaviour}

It is quite important to study the behaviour of the interface between the two fluids, when the oscillations take place. Fig. 3 shows the air-liquid interface patterns at different positions within a complete phase cycle only for water (representing the turbulent scenario) and for PAC $2 \mathrm{~g} / \mathrm{L}$ (representing a laminar flow situation) for brevity. At $\mathrm{t}=0$, the surface of the fluid is subjected to zero parallel shear stresses and when the vertical oscillation starts to act, it induces surface waves on the air-liquid interface and thus enhances undesired dynamic 
mixing behaviour. Ito et al. [10] describe that, the pressure perturbations due to the time dependent fluid acceleration and the viscous/capillary forces due to the fluid-wall relative motion are the two main factors affecting the stability of the oscillating interface.
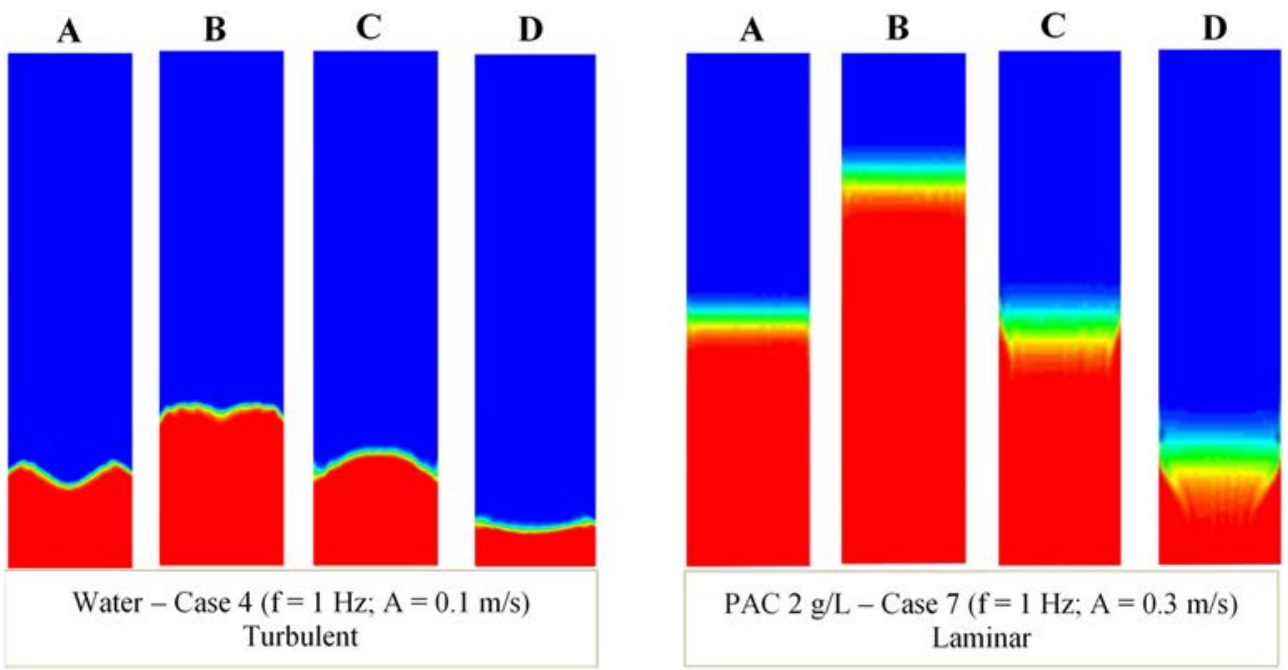

Figure 3: Air-liquid interface patterns two different simulation cases representing both Newtonian and non-Newtonian fluids.

When the interface reached its maximum level, it was strongly impacted by the inertia of the fluid column and the shape of this interface is dependent on the frequency and the velocity amplitude. It could be noted that, the air-liquid interface becomes unstable for the cases with water tending to generate quite a lot of disruptive small peaks like fingers at higher velocity amplitudes. This could be attributed to the surface instabilities generated due to the turbulent flow structure and also due to the absence of surface tension data fed to the simulation. For the laminar cases with non-Newtonian fluids, the air-liquid interface seems to be very calm without any disruptive small peaks. The laminar formulation of the flow structure and the increased viscous resistance could be the reason for such plug-type flow behaviour. However, even the flow is laminar for both non-Newtonian fluids; the interface shows an unstable behaviour at higher velocity amplitudes as shown in Fig. 3. This could be due to the destabilization of the interface by the viscous forces when there is a bulk relative motion between the fluid and the wall. At higher simulated frequencies, the air-liquid interface for both PAC fluids appears to be calmer and tends to show more or less plug type behaviour.

\subsection{Variation of velocity magnitude}

The variation of velocity field within the bulk liquid medium is a better way to understand the impact of the oscillatory motion. The radial velocity profiles illustrated here were extracted along a horizontal line located $200 \mathrm{~mm}$ below the initial air-liquid interface.

\subsubsection{Effect of oscillation frequency}

Fig. 4 shows the dynamic velocity profiles for water, PAC $2 \mathrm{~g} / \mathrm{L}$ and PAC $4 \mathrm{~g} / \mathrm{L}$ fluids at different frequencies and at different positions within a phase cycle for a fixed velocity 
amplitude (Cases 1-5). Note that the velocity values given by Fluent are absolute values, so that stages $\mathrm{C}$ and $\mathrm{D}$ are downwards flow (negative in "real" values).

WATER

PAC $2 \mathrm{~g} / \mathrm{L}$

PAC $4 \mathrm{~g} / \mathrm{L}$

$\multimap-0.25 \mathrm{~Hz}-\square-0.50 \mathrm{~Hz} \neg-0.75 \mathrm{~Hz}-\triangle 1.00 \mathrm{~Hz} \diamond-5.00 \mathrm{~Hz}$
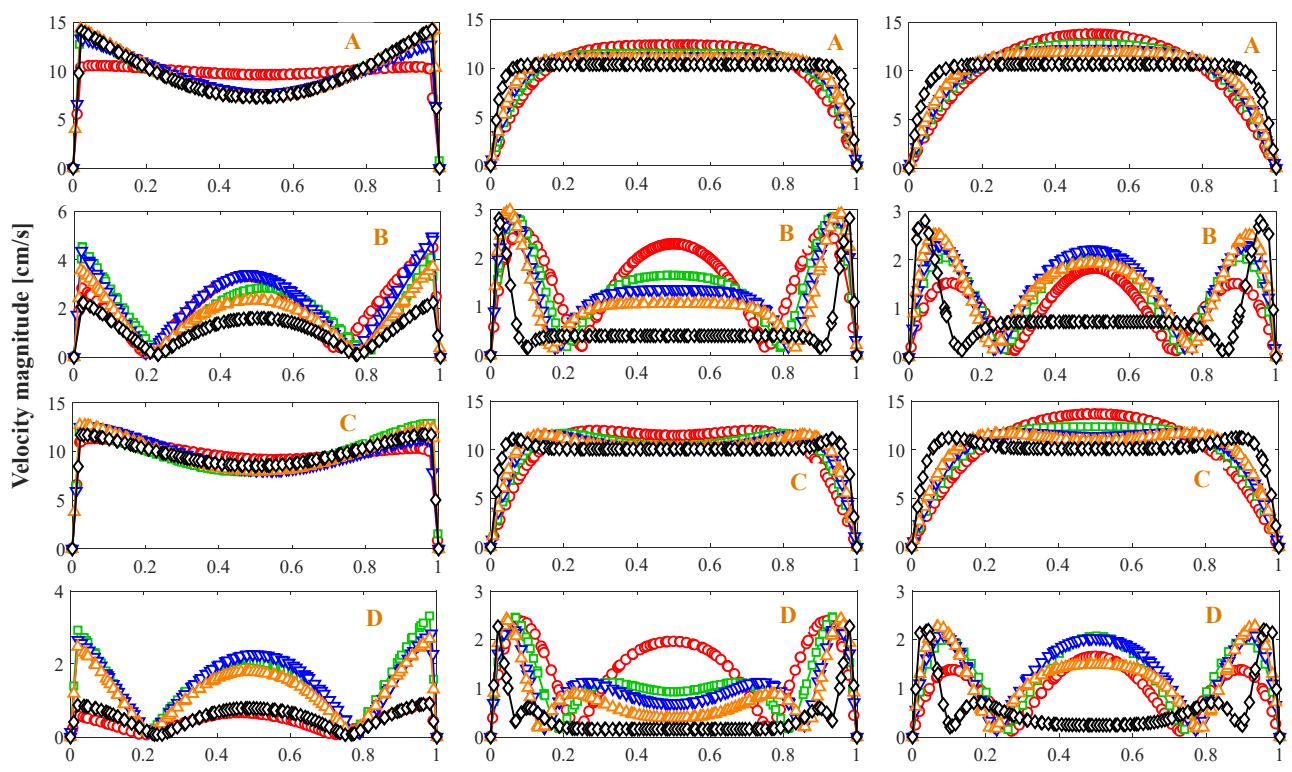

Figure 4: Velocity variation with frequency of oscillation at different phase positions of a complete cycle (velocity amplitude is constant at $0.1 \mathrm{~m} / \mathrm{s}$ ). Non-dimensional horizontal distance (x/D) (-).

The velocity profiles and the corresponding shear rates are calculated in the $\mathrm{x}-\mathrm{y}$ plane between vertical walls. Thus the symmetry line ("pipe centre" for similar 2D cylindrical simulations, $\mathrm{z}-\mathrm{r}$ ) is at the non-dimensional horizontal distance of 0.5 . One should also be aware that since the $(\mathrm{x}-\mathrm{y})$ simulation corresponds more to flow between parallel planes, the calculated wall friction in these simulations would be somewhat different from those obtained with a 2-D cylindrical geometry. Furthermore, in real drilling operations, local uneven geometries that might be present within the well can induce flow instabilities. Similarly, different flow conditions may generate different oscillatory motions to induce flow instabilities. The turbulent behaviour of the flow often occurring within a well makes the scenario more complicated from a rheological point of view when the non-Newtonian fluids are circulating. According to Fig. 4, it could be noted that irrespective of the flow condition whether it is laminar or turbulent, the patterns/shapes of the velocity profiles for all the test fluids at different phase positions are slightly qualitatively similar. Specially, at the phase positions $\mathrm{A}$ and $\mathrm{C}$ for water, the velocity profiles almost coincide each other for all the frequency values. However, there is a considerable variation of the velocity profiles at phase positions $\mathrm{B}$ and $\mathrm{D}$, where the piston is in its maximum and minimum levels respectively. However, when the piston is in the middle position of its way (phase positions A and C), the radial velocity profiles for both non-Newtonian fluids show plug-type behaviour with increased frequency, deviating from the usual parabolic shape. The phase positions B and D 
are the moments where the piston comes to a rest to change its direction and so the bulk liquid medium also lowers its velocity to change the direction. As a result of this, a bell shaped velocity profile could be seen at those phase positions for all the liquids concerned here, where a local velocity maximum (and sometimes a minimum too) can be observed close to the wall region and that propagates towards the centre of the pipe with decreasing amplitude. Furthermore, it is to be noted that, the peak velocity magnitudes achieved by non-Newtonian fluids are lower than that of water, which could be attributed to the higher viscous resistance of the non-Newtonian liquids and also the laminar flow conditions.

\subsubsection{Effect of velocity amplitude}

Fig. 5 shows the radial velocity profiles for water, PAC $2 \mathrm{~g} / \mathrm{L}$ and PAC $4 \mathrm{~g} / \mathrm{L}$ fluids at different velocity amplitudes and at different positions within a phase cycle for a fixed excitation frequency (Cases 4, 6 and 7). Here, again it is to be noted that the velocity values given by Fluent are absolute values, so that stages $\mathrm{C}$ and $\mathrm{D}$ are downwards flow (negative in "real" values).

WATER
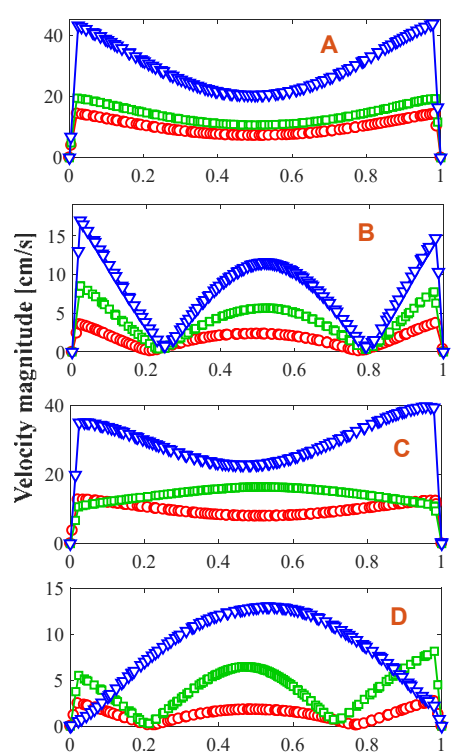

PAC $2 \mathrm{~g} / \mathrm{L}$
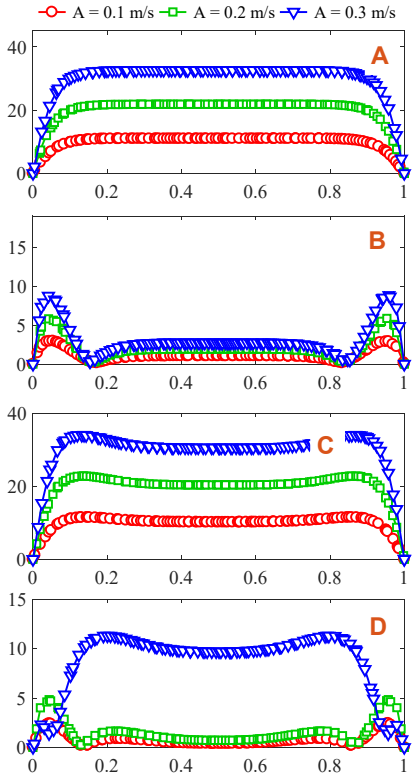

PAC $4 \mathrm{~g} / \mathrm{L}$
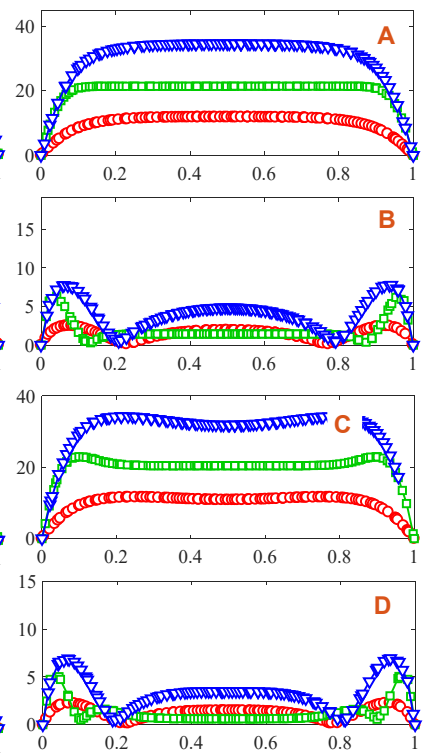

Figure 5: Velocity variation with velocity amplitude at different phase positions of a complete cycle (excitation frequency is constant at $1 \mathrm{~Hz}$ ). Non-dimensional horizontal distance (x/D) (-).

At the first glance, it can be seen that, when the excitation velocity amplitude is increased, the achieved instantaneous velocity magnitudes are also increased for all the three test fluids. That is just because of the increased kinetic energy provided by the excitation via the piston. For almost all the cases, the velocity gradient close to the wall is greater with compared to that within the middle area of the pipe. In the turbulent case for water, the velocity profiles show significant variation in qualitatively and quantitatively with respect to the excitation 
velocity amplitude. However, for the laminar cases in non-Newtonian fluids, the radial velocity profiles just $200 \mathrm{~mm}$ below the air-liquid interface act more likely to be plug-type specially in the phase positions of A and C. Similar to what we observed in Fig. 4, the velocity gradient increases and suddenly changes its sign near the wall region. After the local velocity reaches its maximum, it tends to travel with a very low velocity gradient close to zero for both the PAC fluids. However, it is to be noted that the velocity gradient for water along the horizontal distance is significant and that could be attributed to turbulent effects within the dynamic flow situation. Therefore, it can be depicted that, the effect of driving velocity amplitude on fluids oscillated vertically lead to significant variations in the radial velocity profiles revealing the presence of an alternating upward/downward motion. This complexity is significant in non-Newtonian fluids since these varying velocity magnitudes and hence the velocity gradients change the shear field of the non-Newtonian fluids.

\subsection{Variation of shear rate with frequency of oscillation and velocity amplitude}

Fig. 6 shows the variation of shear rate for some selected simulation cases of PAC $2 \mathrm{~g} / \mathrm{L}$ and PAC $4 \mathrm{~g} / \mathrm{L}$ along the same horizontal line located $200 \mathrm{~mm}$ below the initial air-liquid interface in which the velocity profiles are also drawn. The four cases shown in Fig. 6 have been selected randomly for brevity, just to represent the variation of shear rate. The instantaneous shear rate values along this horizontal position have been calculated using the velocity gradient $\mathrm{dy} / \mathrm{dx}$ as illustrated by Amaratunga et al. [8]. In that sense, water also provides the values for this velocity gradient, but the importance of discussing the variation in shear rate of the non-Newtonian fluids is because, the shear viscosity of such fluids change with the rate of shear.
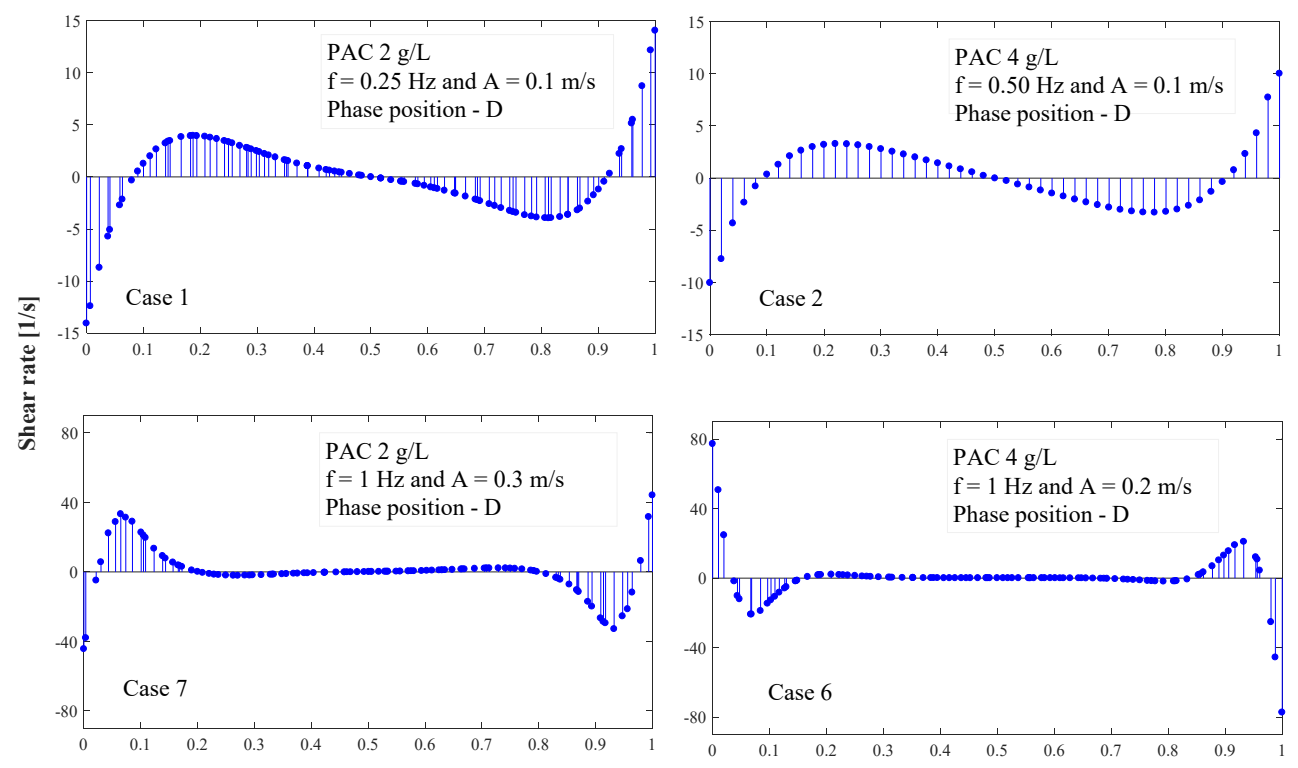

Figure 6: Variation of shear rate along a horizontal line for some selected simulation cases of PAC $2 \mathrm{~g} / \mathrm{L}$ and PAC $4 \mathrm{~g} / \mathrm{L}$. Non-dimensional horizontal distance (x/D) (-). 
The transient results are consistent with the interpretation of the phenomenon, in that the shear rate is maximum when the velocity gradient is maximum and vice versa. Shear rate is normally highest close to the pipe wall in both steady state and transient flows with boundary layer build-up. This fact is greatly proven by the profiles of shear rate shown in Fig. 5 for the phase position D of selected cases. However, it could be noted that, the shear rate variation is somewhat significant even within the centre area of the pipe at low frequencies (see Case 1 and 2 in Fig. 6). But, when the frequency is increased, the shear rate variation becomes more flat and align close to zero within the centre area of the pipe since the flow tends to be more plug-type. The cases illustrated in Fig. 6 depict the wall-induced shear variation as a result of the oscillatory motion experienced by the bulk liquid medium within the pipe. This additional shear introduced by the oscillations causes a variation in shear viscosity due to the shear-thinning property of the fluids. Thus, the superimposition of a sinusoidal oscillatory motion on a bulk liquid medium contained in a pipe leads to a change in apparent local shear viscosity of the shear-thinning non-Newtonian fluids. Consequently, the solids carrying capacities and solids separation capacities of such fluid are altered considerably.

It is worth to check how significant the frequency and velocity amplitudes are in terms of shear rate variation in a vertically oscillating liquid medium. Fig. 7 summarizes the maximum amount of shear rate achieved by the two non-Newtonian fluids for all the simulation cases.

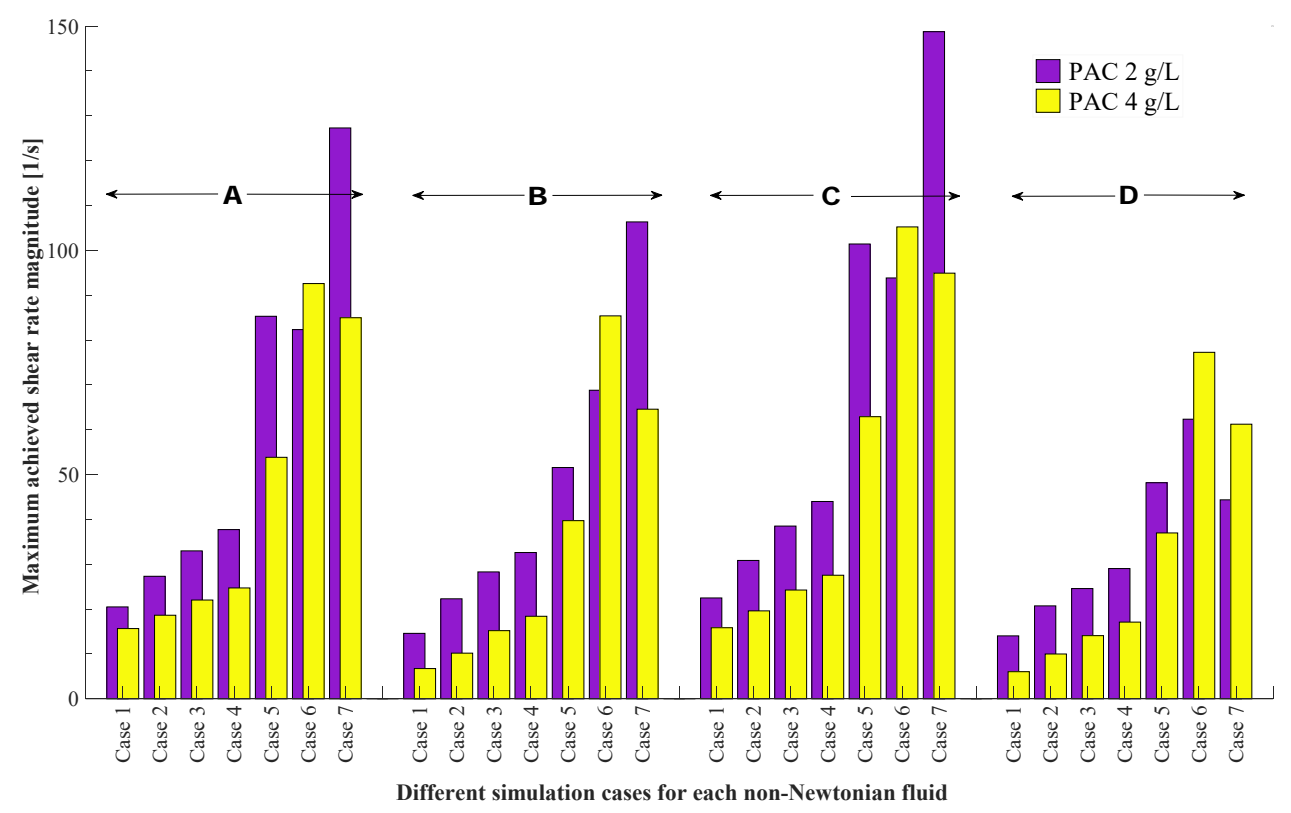

Figure 7: Magnitude of shear rate achieved by the two non-Newtonian fluids for all the simulation cases.

It can be clearly seen from Fig. 7, that for almost all the cases except at higher velocity amplitudes, PAC $2 \mathrm{~g} / \mathrm{L}$ achieves higher shear variation compared to that of PAC $4 \mathrm{~g} / \mathrm{L}$. That could be attributed to the higher viscous resistance of PAC $4 \mathrm{~g} / \mathrm{L}$. Due to the higher viscous resistance, PAC $4 \mathrm{~g} / \mathrm{L}$ is reluctant to be dynamic as PAC $2 \mathrm{~g} / \mathrm{L}$ within the pipe even the frequency is increased. Therefore, the achieved velocity magnitudes and also the velocity 
gradients are lower compared to that of PAC $2 \mathrm{~g} / \mathrm{L}$. However, for the cases with fixed oscillation frequency and higher velocity amplitudes, the increased kinetic energy of the system is more significant than the viscous resistance.

Out of the four phase positions, $\mathrm{A}$ and $\mathrm{C}$ are the levels with highest kinetic energy within the system and that is confirmed by the higher shear magnitudes depicts by the figure. It is obvious that, when the frequency is increased the additional shear induced by the walls are also increased. This indicates that, the tendency of shear field variation is significant at higher oscillation frequencies and higher velocity amplitudes. According to the values shown in Fig. 7, out of all the simulation cases and positions of the phase cycle, let us consider the maximum change in shear rate for PAC $2 \mathrm{~g} / \mathrm{L}$ is approximately $1501 / \mathrm{s}$ and it is in the range of 110 1/s for PAC $4 \mathrm{~g} / \mathrm{L}$ for the concerned geometry. Based on the $\mathrm{K}$ and $\mathrm{n}$ values mentioned in the Table 1 for the power-law modelling of the two liquids, the maximum percentage decrement of the shear viscosity could be calculated to be approximately $14 \%$ for PAC $2 \mathrm{~g} / \mathrm{L}$ and $18 \%$ for PAC $4 \mathrm{~g} / \mathrm{L}$ respectively. It is obvious that, a reduction in shear viscosity for a shear-thinning non-Newtonian fluid means an increment of solids separation rate.

Therefore, it should be paid considerable attention when employing shear-thinning nonNewtonian fluids in applications where they undergo high frequency and high amplitude oscillations because they could namely and greatly affect the non-Newtonian rheology, which eventually alters the solids separation and carrying capacities.

\section{CONCLUSION}

The influence of oscillation frequency and velocity amplitude on dynamic velocity profiles and resulting shear field has been numerically investigated in this study. Seven different cases with varying frequency and velocity amplitudes were simulated using ANSYS Fluent 18.0 version. The air-liquid interface becomes unstable for the turbulent Newtonian cases at higher velocity amplitudes due to the surface instabilities generated by the turbulent flow structure and the absence of surface tension data. For the laminar cases with non-Newtonian fluids, the air-liquid interface seems to be very calm due to the laminar flow behaviour and the increased viscous resistance. When the piston is in the middle position of its way, the radial velocity profiles for both non-Newtonian fluids show plug-type behaviour with increased frequency, deviating from the usual parabolic shape. The peak velocity magnitudes achieved by non-Newtonian fluids are lower than that of water, which could be attributed to the higher viscous resistance of the non-Newtonian liquids and also the laminar flow conditions. When the excitation velocity amplitude is increased, the achieved instantaneous velocity magnitudes are also increased for all the three test fluids. For almost all the cases, the velocity gradient close to the wall is greater with compared to that within the middle area of the pipe. Shear rate is normally highest close to the pipe wall in both steady state and transient flows with boundary layer build-up. However, it could be noted that, the shear rate variation is somewhat significant even within the centre area of the pipe at low frequencies and when the frequency is increased, the shear rate variation becomes more flat and align close to zero within the centre area of the pipe since the flow tends to be more plug-type. According to the calculations based on the results obtained by the simulations, the maximum percentage decrement of the shear viscosity could be approximated as $14 \%$ for PAC $2 \mathrm{~g} / \mathrm{L}$ and $18 \%$ for PAC $4 \mathrm{~g} / \mathrm{L}$ respectively. Therefore, the additional shear introduced by the oscillations causes a variation in shear viscosity due to the shear-thinning property of the fluids, which eventually leads to a change in local shear viscosity of the shear-thinning nonNewtonian fluids. For hydraulic conveying purposes, it can be concluded that the for sinusoidal oscillatory motion of shear thinning liquid medium contained in a pipe leads to an alteration of fluid's solids carrying capacity and solids separation capacity. 


\section{ACKNOWLEDGEMENTS}

Authors gratefully acknowledge the Norwegian Research Council for funding this study under the project "NFR Improved Model Support in Drilling Automation". We are also grateful to Stein-Tore Johansen at SINTEF-NTNU for valuable discussions on CFD simulations.

\section{REFERENCES}

[1] Time, R.W. \& Rabenjafimanantsoa, A.H., On the relevance of laboratory scale rheometric measurements for calculation of complex large scale flows in well drilling and pipe flows. Annual Transaction of the Nordic Rheology Society, 22, pp. 69-79, 2013.

[2] Barnes, H.A., Townsend, P. \& Walters, K., On pulsatile flow of non-Newtonian liquids. Rheologica Acta, 10(4), pp. 517-527, 1971.

[3] Manero, O., Mena, B. \& Valenzuela, R., Further developments on non-Newtonian flow in oscillating pipes. Rheologica Acta, 17(6), pp. 693-697, 1978.

[4] Mena, B., Manero, O. \& Binding, D.M., Complex flow of viscoelastic fluids through oscillating pipes: Interesting Effects and Applications. Journal of non-Newtonian Fluid Mechanics, 5, pp. 427-448, 1979.

[5] Chan, W., Lee, S. \& Liu, C., Effects of frequency and amplitude of oscillation on low Reynolds number pulsating flow in a circular pipe. Engineering Computations, 19(1), pp. 119-132, 2002.

[6] Eesa, M. \& Barigou, M., CFD analysis of viscous non-Newtonian flow under the influence of a superimposed rotational vibration. Computers \& Fluids, 37(1), pp. 2434, 2008.

[7] Torralba, M., Castrejón-Pita, J., Castrejón-Pita, A., Huelsz, G., Del Río, J. \& Ortin, J., Measurements of the bulk and interfacial velocity profiles in oscillating Newtonian and Maxwellian fluids. Physical Review E, 72(1), p. 016308, 2005.

[8] Amaratunga, M., Nybø, R. \& Time, R.W., PIV analysis of dynamic velocity profiles in non-Newtonian drilling fluids exposed to oscillatory motion. Presented at OMAE2018, Madrid, Spain, 17th-22nd June, 2018.

[9] Bird, R.B., Armstrong, R.C. \& Hassager, O., Dynamics of Polymeric Liquids. Vol. 1: Fluid Mechanics, Wiley, 1987.

[10] Ito, T., Tsuji, Y. \& Kukita, Y., Interface waves excited by vertical vibration of stratified fluids in a circular cylinder. Journal of Nuclear Science and Technology, 36(6), pp. 508-521, 1999. 